\title{
A CASE OF ALTERNATING HEMIPLEGIA (MILLARD- GUBLER), WITH REMARKS ON THE PATHS \\ OF THE PUPILLO-DILATOR AND VES- TIBULO-OCULAR FIBERS IN THE BRAIN STEM
}

ANTON LUTZ, M.D.

HAVANA, CUBA

Sharply defined anatomic lesions of the brain stem have recently acquired a new interest in so far as they are now able to furnish us exact information as to the course of those fibers which come from the upper part of the mesencephalon and extend to the centrum ciliospinale. Irritation of these fibers produces psychic as well as reflex dilatation of the pupils. Information is also furnished regarding the path of those other fibers that transmit the irritation from the vestibular apparatus to the oculopontile nuclei. The following case is of interest in this connection.

\section{REPORT OF A CASE}

History.-Felipe de Ll., 31 years old, an engineer, complained Oct. 23, 1918, of reduction of visual acuity. He had always been healthy and strong. There were no family diseases. He denied syphilitic infection. When he was 11 years old, he fell from a height of 3 meters, on his cranium; as a consequence of this accident, there was a small nonadherent cicatrix of the skin in the right parietal region.

Since January, 1918, he had observed indications of metamorphopsia in both eyes, and reduction of his visual power. On June 21,1918 , he suddenly became ill, with symptoms of a left-sided hemiplegia, including the left side of his face, his arm, trunk and leg; at the beginning of this attack he was unconscious for several hours.

Examination.-Examination Oct. 31, 1918, revealed a pale, young, strong, well-nourished man, who complained of headache in the region of the right

fissura centralis. The urine contained a trace of albumin $(0.080 / 00), \frac{1.200}{1.019}$ no sugar and no casts. The Wassermann reaction of the blood was negative. Percussion of the cranium did not produce pain. There was no Romberg sign or symptoms from the cerebellum. The tendon reflexes were normal on the right side, but seemed to be a little diminished on the left side. There were no indications of contracture and no Babinski reflex. The muscular force of the left arm and left leg were undoubtedly reduced, as well as the sensibility. The pupils were equal in both eyes and of medium width; they reacted well to light and accommodation; there were no anisocoria, diplopia, nystagmus, or reduction of the eye movements. Ophthalmoscopically the disks appeared blurred; they showed no prominence, and the entire fundus was covered with small white spots as in albuminuric neuroretinitis. The visual field showed a slight reduction of color sensation in the temporal half of the left eye, being otherwise normal. 
Dec. 23, 1919: The ophthalmoscopic alterations had receded somewhat; otherwise the status was exactly the same as at the time of the previous examination. Examinations of the blood showed negative Wassermann reactions; and many examinations of the urine disclosed no evidence of nephritis. An examination of the cerebrospinal fluid showed a negative Wassermann reaction and a positive globulin reaction (Noguchi), with slight increase in quantity. The original diagnosis of neuroretinitis albuminuria could no longer be maintained. An examination of the vestibular apparatus by Dr. Fernandez Soto showed:

1. Slight reduction of the hearing of the right ear on account of cicatricial otitis (the lesion was caused by an insect in his youth).

2. Both acustici proved to be normal.

3. No spontaneous nystagmus, but spontaneous past pointing of the left arm toward the right. Examination on the turning chair revealed: in different investigations, slight reduction of the amplitude and duration (18 seconds) of the normal nystagmus from the horizontal canals, normal past pointing and vertigo. Ear stimulation with warm water, for the examination of the vertical canals, revealed a complete absence of nystagmus, past pointing, and vertigo; on changing the position of the head (horizontal canals) a reduced nystagmus, past pointing and vertigo appeared. There existed, therefore, a complete inexcitability of the vertical semicircular canals, and hypo-excitability of the horizontal canals. The patient showed no signs of labyrinthine disease (spontaneous noises, vertigo or deafness).

Jan. 21, 1920: On the entire left side of the patient's body (face, arm and leg) a well-marked thermoanesthesia appeared; a few days before he had an attack of dysarthria lasting for some minutes; there was no Babinski reflex.

March 8, 1920: While in the toilet, he suddenly lost consciousness and fell to the floor. He showed a flaccid paralysis of the left arm and left leg (but not of the face) and anesthesia of the entire left half of the body (including the face). The anesthesia could be observed especially well during sleep; one could prick him with a needle on the left side to the point of drawing blood and he showed no reflex defensive movement and did not waken; the reflex defensive movement was marked on the right side. Insensibility extended exactly to the middle of the nose and was most marked when the patient was awake, for temperature and pain and less marked for touch.

March 10, 1920: The patient complained of violent pains and pronounced hyperesthesia in the whole region of the right trigeminus.

March 18, 1920: The patient showed a total peripheral paralysis of the right facialis.

April 17, 1920: Ophthalmoscopically the patient showed in both eyes a well marked choked disk of 3 to 4 diopters. The visual field was normal. The trigeminal pains had disappeared. Vomiting, vertigo, cerebellar symptoms and anisocoria were not present. He had only paralysis of the right facialis and paresis and hypesthesia of the left arm and leg.

April 27, 1920: During the four days before death the patient again suffered pains in the right trigeminus, which increased to such violence that he attempted suicide. On April 27 he felt much better; he arose, and after taking a short walk, he fell, began to perspire copiously, and died within a few minutes with Cheyne-Stokes respiration. Necropsy examination was refused. 
Summary.-A young, strong, nonsyphilitic man, in whom no signs of renal diseases could be traced with certainty, began to complain of metamorphopsia and loss of vision, and showed ophthalmoscopically the appearance of neuroretinitis albuminuria. A complete left-sided hemiplegia (face, arm, leg) receded, leaving only a marked anesthesia of the whole left side, more pronounced for temperature and pain. There were no signs of contracture, Babinski reflex or exaggeration of the tendon reflexes. An examination of the ear failed disclose disease but showed a complete lack of the function of the vertical semicircular canals and a slight reduction of the function of the horizontal semicircular canals. The patient did not have the signs of brain tumor. As time passed complete right-sided paralysis of the facialis and a marked hyperesthesia of the right trigeminus with spontaneous pains developed, added to the left-sided slight disturbance of motility and strong alteration of sensibility. The neuroretinitis albuminuria developed into a typical bilateral choked disk, and the patient died suddenly with symptoms of vagus death.

Necropsy having been refused, cause and localization of the anatomic lesion could not be ascertained with the desired exactness, but we may deduce the following:

The lack of casts in the urine, its normal specific weight and its normal quantity tend to show that a chronic renal malady did not underlie the disease. We have many observations in the earlier ophthalmologic literature showing that the most experienced ophthalmologists pronounced the diagnosis of "nephritis chronica" on account of the fundus picture, while the necropsy failed to discover any disorder of the kidneys but proved the presence of a brain tumor. The whole course of the disease, the developing of a typical choked disk, the positive globulin reaction in the cerebrospinal fluid, the development of a crossed hemiplegia and the sudden death under the symptoms of Cheyne-Stokes' respiration, indicate that we were dealing with a slowly advancing process in the brain stem, probably a tumor, or perhaps a tuberculum.

With greater exactness we can deduce the location of the lesion. The reduction of the sensibility in the whole left side of the body (face, arm and leg) shows an interruption of the lateral fillet between its crossing in the medulla oblongata and its termination in the thalamus opticus; it further shows that this lesion must be located on the right side of the brain stem and higher up than the entrance of the fibers of the trigeminus after their crossing, i. e., in the middle third of the pons. The right-sided peripheral paralysis of the facialis and the right-sided hyperesthesia of the trigeminus indicate, furthermore, a lesion on the right side of the middle part of the pons. The fact that the sensation of pain and temperature alone was completely abolished on the left side of the body and that superficial sensibility was only reduced, together with a peripheral palsy of the facialis without disturbance of the abducens; also the normal functioning of both acustici and the dissociated lesion of the vestibular fibers, and the flaccid character of the left-sided hemiplegia, show that there was a sharply defined anatomic lesion on the right side of the brain stem situated in the upper and external part, limited ventrally by the pontile nuclei, laterally by the vestibular nerve and mesially by the abducens and the mesial fillet; it did not ascend to the floor of the fourth ventricle, because the nucleus of the abducens and the fasciculus longitudinalis posterior were not altered in their function. The lesion included the descending root of the trigeminus (spontaneous pains on the right side 
of the face) and destroyed the lateral fillet, the right pyramidal fibers dispersed between the pontile nuclei and the exit of the right facialis. On the right side the function of the facialis and trigeminus were abolished; on the left side, the greater part of the sensibility and the motility of the arm and leg were abolished. This syndrome has long been known as hemiplegia alternans (type of Millard-Gubler), and it would scarcely be worth while reporting it, were it not for three special points.

\section{ABSENCE OF DEVELOPMENT - OF CONTRACTURE}

Notwithstanding that the patient showed twice, with an interval of about two years, a disturbance of the right pyramid, the paralysis always had a flaccid character and no signs of contracture developed. An interruption of the supranuclear motor path is followed as a rule by a spastic paralysis, and even if it completely recedes, an exaggeration of the corresponding tendon reflexes remains, and a positive Babinski reflex develops. The signs of contracture develop at the earliest after fourteen days, but as a rule from one to three months after the attack, and they fail to appear only in the rarest exceptions. Explanation of the developing of the cerebral contracture has been attempted, and careful investigations have shown the interruption of the pyramidal tract and the integrity of the posterior roots to be the essential conditions. Both conditions were present in my case, and nevertheless the reflexes of the left side were rather diminished. Of the different theories, that of van Gehuchten is best suited to the present case; it holds that the contracture is caused by the continuance of the functioning of those fibers which connect the pontile nuclei with the cerebellum (fibrae cortico-ponto-cerebello-spinales) through the middle cerebellar pedunculus.

\section{LACK OF LESION OF THE PUPILLO-DILATING FIBERS}

During the whole course of the disease I paid special attention to the observation of the pupillary reaction in order not to overlook the appearance of anisocoria. We know from many observations that a lesion of the brain stem is sometimes followed by a homolateral miosis, and therefore lesions of the brain stem are apt to illustrate to us the exact topographical course of the pupillary fibers. If we wish to determine their situation in the brain stem, we must look for the following points: $(a)$ height and latitude of the lesions, and $(b)$ crossing of the fibers.

(a) During the entire course of the disease both of the patient's pupils were equal and of medium size. This agrees with other symptoms that indicate a lesion in the tegmentum of the pons not having reached the floor of the fourth ventricle. Déjerine became convinced, through his anatomic investigations, that the sympathetic fibers run through 
the upper layers of the substantia reticularis, medially of the root of the trigeminus and laterally of the middle of the brain stem. In a previous article I expressed the opinion ${ }^{1}$ that the sympathetic fibers are responsible for the pupillo-dilatation and that they run down from the mesencephalon to the centrum cilio-spinale, together with the fasciculus longitudinalis posterior which is situated just on top of the substantia reticularis. Spiller ${ }^{2}$ gave some further instances showing that the lesion of the brain stem can be followed by a homolateral miosis, and in his cases the sympathetic fibers have been interrupted by foci of destructive nature. In consideration of this point, tumors like gliomas which entangle the fibers are less apt to give information than tuberculous foci that destroy them. As we have not yet many observations of lesions of the brain stem which especially consider the localization of the pupillo-dilating fibers, it may be well to cite here, two further examples illustrative of the subject. F. Raymond ${ }^{3}$ gives an exact description of a case closely resembling my own: Paralysis of the facialis and of the abducens on the left side, and spastic paresis of the right arm, trunk and leg, together with cessation of all sensibility on the right side of the body (face, arms, trunk and leg) occurred; the patient showed no anisocoria. Another patient ${ }^{4}$ who had, on the right side, hemiparesis and hypesthesia of the arm, trunk and leg, and on the left side a paralysis of the facialis, abducens and masseter, together with anesthesia of the left side of the face, had a miosis without alteration of the pupillary reaction on the left side. The fact that the masseter was paralyzed shows that the lesion extended higher up in the level and extended more medially than the previously reported case and my own.

We may therefore conclude that the lesion of the brain stem will be accompanied by a homolateral miosis only when it reaches the highest and medial part of the substantia reticularis.

(b) Most authors believe, with me, that in man the sympathetic fibers do not undergo a crossing between the mesencephalon and the centrum cilio-spinale; some question that they undergo any crossing at all.

In favor of the first opinion that the pupillo-dilation fibers running down from the mesencephalon to the centrum cilio-spinale (which is

1. Lutz, A.: The Light Pupillary Reflex, Its Path and Its Abolition Called Immobility of the Pupil to the Light Reflex, and Report of a Case of Unilateral Argyll Robertson Pupil in which Consensual Reaction Existed in Both Eyes, Arch. Ophth. 47:266 and 370, 1918.

2. Spiller: The Oculo-Pupillary Fibers of the Sympathetic System. Am. J. M. Sc. 100:325, 1920.

3. Maladies du systeme nerveux, 1900, p. 63.

4. Maladies du systeme nerveux, 1903, p. 436. 
the homologue of the motor cells of the anterior horns for the pyramidal system) remain uncrossed on the brain stem, are the above named reports on anotomic lesions, as well as the experiments of Levinsohn. ${ }^{5}$ Levinsohn cut the brain stem and the medulla-spinalis cervicalis in rabbits on one side and observed that this lesion was followed by a homolateral miosis. This miosis was always homolateral and not pronounced, but unmistakable, when he made the cut through the medial parts of the brain stem above the trigeminal entrance. Miosis was stronger and mostly homolateral, but sometimes also in a minor degree heterolateral after he had made the cut below the entrance and more lateral (descending roots of the trigeminus) miosis was never accompanied by a reflex immobility of the pupil. This developed only when he made the cut at a height between anterior and posterior corpora-quadrigemina. Levinsohn explained his experimental miosis by way of irritation of the pupillo-constrictor fibers, instead of by way of paralysis of the pupillo-dilating fibers. Therefore Levinsohn found in rabbits, as did Karplus and Kreidl ${ }^{\circ}$ in cats, that each half of the cervical cord conducts impulses from the brain stem to both cervical sympathetic nerves. In man, however, all our experience tends to show that the miosis is homolateral with a lesion of the brain stem. This shows us that the important experiments of Karplus and Kreidl made on cats and apes with regard to the subthalamic dilation center and its connection cannot be applied unreservedly to human pathology.

The question of crossing is more difficult to decide in the parts of the pedunculus situated higher up because of the lesion occurring at the same time in the fibers of the oculomotorius responsible for pupilloconstriction. For the following reasons we may conclude that they do not undergo crossing below the anterior quadrigemina: Brissaud ${ }^{7}$ remarks that an ophthalmoplegia externa pura is as a rule accompanied by a miotic pupil without alterations of the reactions. Cases of unilateral Argyll Robertson pupil, such as are reported by Tanzi, ${ }^{8}$ Salomonsohn, ${ }^{9}$ and myself, ${ }^{10}$ show miosis. The results of Levinsohn's experiments with rabbits, and cases of hemoplegia reported by Spiller, and the ease of unilateral thalamic lesion of Ingham, referred to by Spiller in the same article, apparently point to this conclusion.

5. Beiträge zur Physiologie des Pupillarreflexes, Von Gräefes Arch. f. Ophth. 59:191, 1904.

6. Karplus and Kreidl : Arch. f. d. ges. Physiol. 129:138, 1909; 1910, p. 401 ; 1912, p. 109.

7. Maladies du systeme nerveux, 1895.

8. Riv. di patol. nerv., 1899.

9. Von Gräefe's Arch. f. Ophth. 54:211, 1902.

10. Arch. of Ophth. 47:266, 1918. 
If we deduce from the foregoing that the pupillo-dilation fibers do not cross, in man, between corpus quadrigeminum anterius and centrum cilio-spinale, we are forced to search for the crossing higher up. For it is a general anatomico-physiologic law that every efferent motor system undergoes a crossing in its supranuclear path. We must further remember that there exists a direct, as well as a consensual, pupillodilating reflex in man; this indicates that a partial decussation of the pupillo-dilation fibers must occur higher up, and it is quite possible that this happens in the dorsal tegmental decussation of Meynert. It appeared logical to look for the crossing of the sympathetic fibers in the commisura cerebri-posterior, which Ramon y Cajal considers an optic reflex path; but we know, through the experiment of Harris, ${ }^{11}$ that its cutting is not followed by miosis, and we must therefore conclude that at least the majority of the pupillo-dilating fibers do not take this course.

The question of where the sympathetic fibers have their source is as yet unsolved. Histologic researches of Ramon y Cajal show that the fasciculus bigeminalis connects the lenticular region with the corpora quadrigemina anteriora. Ramsay Hunt ${ }^{12}$ came to the conclusion that these fibers of the ansa peduncularis originate exclusively from the globus pallidus, i. e., from those parts of the nucleus lenticularis which, in the realm of motion, plays the same important rôle, through control and regulation of automatic and associated movements, as the thalamus opticus in the domain of sensation, and both of which lost phylogenetically their early importance with the development of the cortex cerebri. Testut tells us that part of the ansa peduncularis enters the fasciculus longitudinalis posterior, which has perhaps for the basal motor ganglions the same importance as the pyramis for the cortical motor region. These pallidal fibers govern the principal movements of Munk, as do the pyramidal fibers with Munk's separate movements. Ramsay Hunt concluded that the fibers originating in the globus pallidus have a purely motoric function: this means that those which run down in the fasciculus longitudinalis posterior dilate the pupil. Consequently, their interruption would be followed by miosis.

Karplus and Kreidl ${ }^{\circ}$ determined by experiments with cats and apes, a center for pupillo-dilation, situated in the hypothalamus, near the dorsomedial portion of the foot of the cerebral peduncle, in the frontal part of the corpus subthalamicum. They could further show that the

11. Harris: Binocular and Stereoscopic Vision in Man and Other Vertebrates with Its Relation to Decussation of the Optic Nerves, the Ocular Movements and the Pupil Light Reflex, Brain 27:107, 194.

12. Hunt, Ramsay: Progressive Atrophy of the Globus Pallidus, Brain, 40: 58, 1917 . 
irritation of this center in apes had a stronger effect on the heterolateral sympathetic cord. Their center corresponds, probably, to the corpus of Luys, limited beneath by the pedunculi cerebri, medially by the substantia grisea of the third ventricle, above by the thalamus opticus and laterally by the capsula interna. Ramsay Hunt considers it a derivative of the globus pallidus. All the subthalamic centers undergo, after the histologic search of Ramon y Cajal, a partial crossing; the fibers of the corpus of Luys cross just in front of the red nucleus (commissura de Forel). Ramsay Hunt also came to the conclusion that a degeneration of all the fibers originating in the large cells of the globus pallidus is followed by a syndrome of paralysis agitans, i. e., rigidity, tremor and loss of the automatic function of the corresponding somatic muscles. Another peculiarity of the Argyll Robertson pupil is that it remains not only immobile to light, but also loses the constant and automatic small oscillating movements which the Germans call "Pupillenunruhe"; the amaurotic pupil is also immobile on light incidence but never loses its "Pupillenunruhe," nor becomes miotic. The fact that an Argyll Robertson pupil is not influenced by a retinal process, e. g., that it remains miotic, although the patient becomes blind later by optic atrophy, indicates clearly that the Argyll Robertson pupil is not only produced by interruption of the afferent optico-sensoric path, but by still other etiologic factors. Furthermore, the Argyll Robertson pupil not only grows stiff, but its dilatation becomes difficult by reason of atropin. One can therefore consider the possibility that the peculiar stiffness of the Argyll Robertson pupil is caused by an interruption of striospinal fibers traveling in the fasciculus bigeminalis and their continuance in the fasciculus tecto-spinalis. Against such acceptance, we must say that bulbar as well as spinal niosis are as little followed by rigidity of the pupil as the amaurotic light immobility.

Nevertheless, we must accept the fact that the peculiar stiffness of the Argyll Robertson pupil is produced by a lesion of the striothalamo spinal system, because rigidity plus paralysis are as typical for lesions of the globus pallidus as is spastic paralysis for lesions of the pyramid. Perhaps it is produced by interruption of those fibers which enter in connection with the deepest layers of the corpora quadrigemina-anteriora. We may also remember here that we not seldom find in old people miotic and rigid pupils without loss of light reflex; this is perhaps caused by senile vascular changes in the lenticular region, whereas a lesion in the dorsal tegmental decussation conduces to the Argyll Robertson pupil, namely: to loss of light influence through interruption of the tectobulbar fibers, ${ }^{1}$ to miosis through interruption of the tectospinal fibers, to a rigidity through interruption of the 
pallidal fibers. If these deductions are correct, we are forced to accept that the crossing of the latter two fibers takes place higher up in Meynert's crossing than the partial decussation of the first named fibers. Also, we will find the-following homology between the paths for pupillo-constriction and pupillo-dilation:

\section{Path for Pupillo-Dilation Path for Pupillo-Constriction}

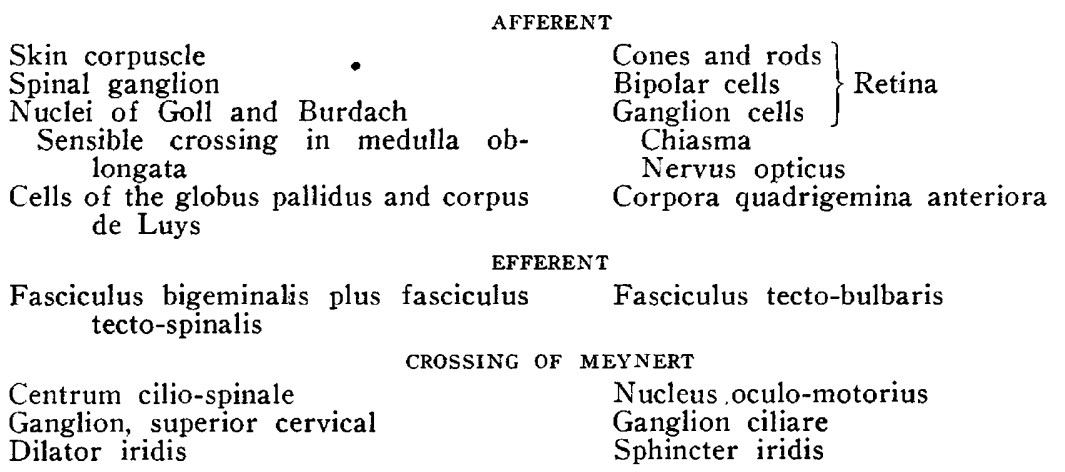

DISSOCIATION OF THE FUNCTION OF THE SEMICIRCULAR CANALS

The patient does not show any signs of disease of the nervii acustici, but there is doubtless a dissociation of the function of the semicircular canal. The examination after the method of Baranyi, with warm water, discloses that the vertical canals do not react, either with nystagmus, vertigo, past-pointing or nausea; whereas the horizontal canals react in every way, though in a lesser degree, as shown by the reduced amplitude and the reduced duration of the experimentally provoked nystagmus. Such a dissociation of the semicircular canal can be caused, according to experience, only by a lesion of the brain stem. We know, through the systematic investigation of Jones, ${ }^{13}$ Mills and Weisenburg, that the vertical and the horizontal semicircular canals send their fibers through different paths to the bulbar nuclei, to the cerebellum and to the cortex. The horizontal canal fibers divide before the magnocellular Deiter's nucleus, and send their cerebellar fibers, which correspond to the unconscient muscular fibers of the body, through the inferior cerebellar peduncle; the vertical canal fibers go higher up into the pons (perhaps to the triangular nucleus of Obersteiner) and send their cerebellar fibers through the middle cerebellar peduncle. Our patient lacks from the vertical semicircular canals, not only nystagmus caused by the fibers which go to the ocular nuclei, but also vertigo caused by the fibers which ascend together with the conscient muscular sense and superficial sensibility through the medial laqueus to the cortex,

13. Jones: Equilibrium and Vertigo, Philadelphia, J. B. Lippincott Co., 1919. 
and which produce the sensation of vertigo by the difference of the conscient muscular sensation and the unconscient cerebellar musculartonus. The patient shows further a lack of normal past-pointing produced by the fibers which go to the cerebellum and cause the unconscient muscular-tonus. It must, therefore, be accepted that the fibers of the vertical semicircular canals are blocked in their totality. It would be quite easy to explain this loss of function if it were found only on one side, because all the other symptoms indicate anatomic lesion in the middle story of the pons just in the neighborhood of the entrance of the nervtus vestibularis. Such a unilateral loss has been clinically observed by Jones ${ }^{13}$ in a man who, according to the opinion of Weisenburg, suffered from a typical lesion of the middle cerebellar peduncle: the left vestibularis was completely normal; the right one showed only the loss of function of the vertical semicircular canal. The patient in my case showed the loss of function on both sides, and the above deduced anatomic lesion cannot explain directly the loss of the second vestibularis, as we must expect from the cerebellar fibers that they ascend through the homolateral middle cerebellar peduncle. The only manner in which we can explain such bilateral loss is the following: The vertical fibers ascend higher up in the pons and run, therefore, a longer way beneath the floor of the fourth ventricle than the more numerous horizontal fibers; by their longer course they are more exposed to a blocking through an increase of the intraventricular tension. Already Wells P. Eagleton ${ }^{14}$ has pointed out that a reduced excitability of the vestibular apparatus of the apparently healthy side is produced by an increase of intracranial pressure. Jones came to the same conclusion as a consequence of numerous careful investigations. Both could observe that a decompressive operation reestablished the function of the vertical semicircular canals. An excellent illustration is one case observed by Jones: ${ }^{13}$ An apparently healthy man complained of headache; an examination of the vestibular apparatus revealed loss of the function of the vertical semicircular canals on both sides; irritation of the horizontal canal produced nystagmus but no vertigo; both acustici were normal and repeated neurologic examinations were negative. Some days later the patient died and necropsy disclosed abscess of the fourth ventricle. The exact investigation of the vestibular apparatus dates back only several years, since its introduction by Baranyi; we have, therefore, only a few observations on

13. Jones: Equilibrium and Vertigo, p. 124.

14. Eagleton, Wells P.: Decompression for the Relief of Disturbances of the Auditory Apparatus of Intracranial Origin, Laryngoscope 23:592 (May) 1913.

13. Jones: Equilibrium and Vertigo, p. 58. 
dissociation of the vestibular function. Jones, however, found thirtyeight among 700 cases on which he made clinical investigations, which perhaps indicates that in the near future numerous similar observations will be made. In all his cases the acustici were normal, the horizontal canals reacted, and the vertical canals showed a partial or complete unilateral or bilateral loss of function. We are not yet able to interpret exactly its bilateral loss. Eagleton, as well as Jones, could also have made the observation that tumors of the cerebellopontile angle block very early, through pressure on the brain stem, but only the function of the vertical semicircular canals of the opposite side is affected.

Therefore, the observation of the eyegrounds with a transition of neuroretinitis albuminuria in a typical choked disk, as well as the bilateral loss of function of the vertical semicircular canals, indicate that the patient in the present case suffered from an increase of intracranial pressure, which probably was produced by an intrapontile tumor causing a crossed hemiplegia and leaving intact the sympathetic pupillo-dilating fibers, because the lesion did not ascend high enough into the substantia reticularis.

I take pleasure in thanking Dr. Fernandez Soto and Prof. José Valdes Anciano for kindly placing literature at my disposal. 